\title{
Spreading Depression Increases Immunohistochemical Staining of Glial Fibrillary Acidic Protein
}

\author{
Richard P. Kraig, ${ }^{1}$ Liming Dong, ${ }^{2}$ Ronald Thisted, ${ }^{2,3}$ and Christine B. Jaeger ${ }^{4}$ \\ ${ }^{1}$ Departments of Neurology and Pharmacological and Physiological Sciences, ${ }^{2}$ Department of Statistics, and ${ }^{3}$ Department \\ of Anesthesia and Critical Care, The University of Chicago, Chicago, Illinois 60637 and ${ }^{4}$ Center for Paralysis Research, \\ Department of Anatomy, School of Veterinary Medicine, Purdue University, West Lafayette, Indiana 47907
}

\begin{abstract}
Reactive astrocytosis is a process by which astrocytes respond to brain injury by showing an increase in glial fibrillary acidic protein (GFAP) staining that is associated with hypertrophy and/or hyperplasia of these cells. Because spreading depression (SD) is a perturbation uncomplicated by neuronal necrosis and is seen in both in vivo and in vitro neural structures, we sought to determine whether SD was a sufficient stimulus to induce enhanced GFAP staining. SD was elicited in anesthetized rats by application of $\mathrm{KCl}$ to parietal cortex for $3 \mathrm{hr}$; equimolar $\mathrm{NaCl}$ was applied to contralateral cortex. SD was confirmed by monitoring DC potentials in frontal neocortices. Animals were allowed to recover for $48 \mathrm{hr}$, and their brains were processed for semiquantitative and computer-based analyses of GFAP staining intensity. Experimental GFAP staining was referenced to contralateral control levels. Neocortical SD (13-37 SDs) was associated with a significant $\left(p<10^{-4}\right), 43 \%$ increase in GFAP staining intensity, which remained statistically greater than normal for more than 2 weeks. If SD was inhibited by combined hyperoxia and hypercarbia, only a nonsignificant $(p>0.20), 7 \%$ increase in GFAP staining was seen. Thus, SD may be a useful physiologic process with which to begin to explore the cellular mechanisms that induce the transformation of normal astrocytes into reactive species.
\end{abstract}

The cytoskeleton consisting, in part, of intermediate filaments is intimately involved in structural and functional changes within cells (Niggli and Burger, 1987). Because astrocytes are among the most dynamic of brain cells, with profound capacities for anatomical and physiologic change (for review, see Kimelberg and Ransom, 1986; Norenberg et al., 1988), concomitant alterations in their principal intermediate filament constituent, glial fibrillary acidic protein (GFAP), can be expected. Indeed,

\footnotetext{
Received Oct. 1, 1990; revised Feb. 15, 1991; accepted Feb. 25, 1991.

This study was supported by The National Institute of Neurological Disorders and Stroke (NINDS) Grant NS-19108, by an Established Investigator Award from the American Heart Association, by The Lucille P. Markey Charitable Trust, and by University of Chicago Brain Research Foundation funds to R.P.K. C.B.J. was supported by U.S. Public Health Service Grant NS-27694 from NINDS. We thank Mrs. Sally Schlessinger for assistance with image analyses, Mrs. Marcia Kraig for assistance with animal experiments, and Mrs. Jing $\mathrm{Li}$-Du for preparing the immunohistological slides.

Correspondence should be addressed to Richard P. Kraig, Department of Neurology, Box 425, The University of Chicago, 5841 South Maryland Avenue, Chicago, IL 60637.

Copyright (C) 1991 Society for Neuroscience $0270-6474 / 91 / 112187-12 \$ 03.00 / 0$
}

transformation by injury of normal astrocytes into so-called "reactive species" is associated with increased immunohistochemical staining for GFAP, a change that is recognized as one of the histological hallmarks of this metamorphosis (Bignami and Dahl, 1976; Latov et al., 1979). The two other histological defining characteristics of reactive astrocytosis are hypertrophy and hyperplasia (Duchen, 1984). These latter two phenomena might account for an increase in GFAP staining because of an associated increase in the size and the number of GFAP-positive cells. However, recent evidence indicates that at least two forms of increased GFAP staining can be seen after brain injury. For example, after global ischemia, astrocytic hypertrophy and increased GFAP staining are transiently seen for a few weeks in brain regions where few neurons are irreversibly injured (Petito et al., 1990). On the other hand, hypertrophy and hyperplasia of a more lasting duration occur along with increased GFAP staining in hippocampal regions where neurons progressively die over days after ischemia (Petito et al., 1990).

Little is known about the mechanisms that govern the induction of reactive astrocytosis after ischemia. Spreading depression $(\mathrm{SD})$ is a propagating phenomenon that is associated with a transient loss of membrane potential and electrophysiologic activity in brain cells of susceptible neural tissues (Bures et al., 1974). It can be elicited in vivo (Bures et al., 1974) as well as in vitro in isolated brain slice preparations (Snow et al., 1983). SD causes many physiologic and metabolic changes that are similar to those seen during ischemia. Furthermore, recurrent SDs do not result in any irreversible neuronal injury as evidenced by hematoxylin and eosin staining (Nedergaard and Hansen, 1987). Thus, SD may be an opportune, physiologic perturbation with which to begin to study the mechanisms by which astrocytes are transformed into reactive species.

In this study, we addressed the issue of whether or not SD was a sufficient perturbation to initiate increased staining of GFAP. Our measurements demonstrate that recurrent neocortical SD is associated with a highly significant, but transient, increase in GFAP staining that is absent if SD is prevented.

Portions of these results have been reported in preliminary form (Kraig and Jaeger, 1990).

\section{Materials and Methods}

Animal preparation and recording. Nonfasted male Wistar rats (24 animals, $275-450 \mathrm{gm}$ ) were anesthetized with halothane (5\% induction, $3 \%$ during surgery, and $1.0-1.5 \%$ during electrophysiologic recordings) and allowed to breathe spontaneously a $30 \% \mathrm{O}_{2} / \mathrm{N}_{2}$ mixture. The halothane dose was adjusted to keep arterial carbon dioxide tension $\left(\mathrm{PaCO}_{2}\right)$ within 55-70 torr during recording periods to provide a level of anesthesia that resulted in no change in heart rate, blood pressure, or with- 
drawal movement when a paw was pinched with forceps. A tail artery was cannulated to monitor arterial blood pressure and to sample arterial blood. Small (1-2 mm-diameter) craniotomies were made $2 \mathrm{~mm}$ lateral to and on both sides of the sagittal suture $3 \mathrm{~mm}$ anterior to bregma and $5 \mathrm{~mm}$ posterior to bregma. Latex tubing ( $5-\mathrm{mm}$ inner diameter) cut to a height of $2 \mathrm{~mm}$ was glued to the skull with cyanoacrylate to create superfusion wells around the left and right posterior craniotomies. Animals were mounted in a standard stereotaxic frame and superfused with a physiological Ringer's solution containing (in $\mathrm{mM}$ ) $\mathrm{Na}^{+}, 143.5$; $\mathrm{K}^{+}, 3.0 ; \mathrm{Ca}^{2+}, 1.5 ; \mathrm{Mg}^{2+}, 1.5 ; \mathrm{Cl}^{-}, 115 ; \mathrm{HCO}_{3}{ }^{-}, 26.4$; gluconate, 9.6; sucrose, 8.0 ; and glucose, 5.0 ; this mixture, when aerated with $5 \%$ carbon dioxide and $95 \%$ oxygen, had a $\mathrm{pH}$ of $7.30-7.40$ (modified from Bretag, 1969). The exposed dura was retracted in each of the four craniotomics.

A glass micropipette (filled with $150 \mathrm{mM} \mathrm{NaCl}$; tip diameter, $2-4 \mu \mathrm{m}$ ) was positioned $500 \mu \mathrm{m}$ below the pial surface of the left and right anterior craniotomies to monitor DC signals from each neocortex. Microelectrodes were connected to an Axoprobe A-1 amplifier system (Axon Instruments, Burlingame, CA), and DC signals were displayed on a chart recorder. An indifferent electrode filled with $1 \mathrm{~m} \mathrm{KCl} 3 \%$ agar was positioned on the left temporalis muscle.

Arterial $\mathrm{pH}$, oxygen tension $\left(\mathrm{PaO}_{2}\right)$ and $\mathrm{PaCO}_{2}$ were monitored with a Corning 168 blood gas analyzer (Ciba Corning Diagnostics Corp., Medfield, MA) throughout electrophysiologic recording periods. Blood glucose was measured with a glucometer (Miles Laboratories, Naperville, IL). When blood gas variables were stabilized, recurrent SDs were induced in the left neocortex by filling the posterior superfusion well with a liquified (i.e., $38-40^{\circ} \mathrm{C}$ ) $3 \%$ agar gel of $1 \mathrm{~m} \mathrm{KCl}$. The right superfusion well was filled with a $3 \%$ agar gel made with $1 \mathrm{M} \mathrm{NaCl}$. In later experiments (see Results), $3 \mathrm{M} \mathrm{KCl}$ and $\mathrm{NaCl}$ agar gels were used. Cortices were exposed to these gels for $3 \mathrm{hr}$, and then the wells and electrodes were removed, skin incisions were closed with stainless steel staples, and the animals were allowed to recover. Within 2-3 $\mathrm{hr}$ of recovery from anesthesia, animals displayed feeding, drinking, and grooming behavior typical of that seen before experimental manipulations. A total of 15 animals were included in this experimental group.

A second group of animals $(n=6)$ were prepared as described above except that, after DC-recording electrodes were implanted into frontal neocortices, animals were exposed to combined hyperoxia and hypercarbia before SD was initiated. Specifically, inhaled gasses were first altered to $100 \% \mathrm{O}_{2}$, and then 5-10 min later, $\mathrm{CO}_{2}$ was slowly added and adjusted to kecp $\mathrm{PaCO}_{2}$ betwecn 100 and 120 torr (Gardner-Medwin, 1981). Inspired halothane remained constant at $1-2 \% . \mathrm{KCl}$ and $\mathrm{NaCl}$ gels were then applied to neocortices, and animals were processed as described above. Finally, a third group of animals $(n=3)$ were prepared as above except inspired gasses were altered to $100 \% \mathrm{O}_{2}$ before $3 \mathrm{M}$ saline gels were applied to cortices.

Immunohistochemistry. Two days after exposure to recurrent SD, animals were reanesthetized with halothane and killed by perfusionfixation using $4 \%$ paraformaldehyde in $0.1 \mathrm{M}$ phosphate buffer ( $\mathrm{pH} 7.4$ ). Brains were removed, postfixed for $2 \mathrm{hr}$, placed in $0.15 \mathrm{M}$ phosphate buffer (pH 7.4) overnight, and stored in $30 \%$ sucrose and $0.1 \mathrm{M}$ phosphate buffer (pH 7.4) for 2-7 d. Sucrose-infiltrated brains were frozen in dry ice and cut into $30-\mu \mathrm{m}$ coronal sections. Nine sections spaced 1 $\mathrm{mm}$ apart and, beginning $3 \mathrm{~mm}$ anterior to bregma, were taken from each brain and stained for GFAP using the peroxidase-anti-peroxidase method (Jaeger, 1988) and a polyclonal antibody (22522, Incstar Inc., Stillwater, MN). Other brains from normal animals $(n=4)$ were similarly stained for GFAP.

Image analysis. A computer-based image analysis system (Bioquant IV, R \& M Biometrics, Nashville, TN) was used to quantitate the relative (left, experimental, versus right, control) neocortical GFAP staining intensity and area (Fig. 1). Brain sections were transilluminated with a 30-mm miniature flood lamp, and images of individual sections were captured with a charge-coupled camera (CCD-72S, Dage MTI, Michigan City, IN) using a $200-\mathrm{mm}$ zoom lens. The light source and camera were connected to line voltage via a Sola constant voltage transformer (Sola, Elk Grove Village, IL) to reduce voltage fluctuation-induced variations in image intensity. Monochrome images of brain sections were digitized with a Targa M8 graphics adapter (Truevision, Inc., Indianapolis, IN) at a resolution of $518 \times 482$ pixels and a 256 -shade gray scale using an AST 386 computer (AST Research, Inc., Irvine, CA). The image analysis protocol for each brain section, modeled after that described by Mize et al. (1988), is depicted in Figure 2 and consisted of the following steps: (1) Transillumination light intensity was adjusted to a level needed to visualize a given brain section and left constant throughout image capture and processing steps. (2) A blank, "coverslipped" microscope slide was transilluminated, and the resultant image was stored for subsequent background correction of the brain section-related captured image. (3) A gray scale range was determined for the brain section, which began at a threshold value where only the darkest neocortical GFAP staining was detected and ended at a level where registration of neocortical GFAP staining reached saturation (Fig. 2, top). This range was divided into six equally sized gray scale increments, and the area measured within each increment was weighted to reflect differences in staining intensity (Fig. 2, bottom). The darkest area was multiplied by 6 , and each progressively lighter increment was multiplied by $5,4,3,2$, or 1 , respectively. Weighted areas were then added together, and the resultant sum was normalized by dividing this value by the total area measured for a given cortical side. The normalized sum from the experimental cortex was divided by a similar value from the contralateral control cortex. Thus, nine relative and normalized GFAP staining ratios (one per brain section) were produced for each experimental animal.

Statistical methods. GFAP staining ratios of 1 indicated no difference between left and right sides. Staining ratios greater than 1 indicated that recurrent SD increased experimental GFAP staining on the left above that of the contralateral neocortex. Staining ratios of 1 indicated no difference. These ratios were converted to natural logarithms so that 0 corresponded to no difference between cortical sections, and so that a $t$ test could be used to determine if differences of logarithms varied significantly from 0 . Rat-to-rat variability was comparable to variability between brain sections within rats. As a result, treating each section as an independent response was not appropriate. Data from individual sections were averaged to yicld a single number for cach rat, and these mean log-ratios were used in $t$ tests. In the text, we report changes in log-ratios as percentage changes. Two-sided $p$ values are reported.

Image-analysis-system characteristics. Before data acquisition from experimental brain sections was begun, the characteristics of the image analysis system were defined. Gray scale linearity was verified by assessing $(n=5)$ average field light intensity (measured in lux) with a corresponding gray scale value. Kodak wratten neutral density filters (Kodak, Inc., Rochester, NY) were used to reduce light incident on the video camera in defined steps $(0.1-1.2$ optical density units). This range of filters was linearly related (correlation coefficient, $r=0.980$ ) to a gray scale range of 4-254. Brain sections were imaged over a gray scale range of 60-120, well within the linear range of the system. System reproducibility was determined by repeatedly $(n=10)$ measuring the staining area and intensity of a single brain section. The avcrage and standard deviation for the ratio (left, experimental, over right, control) of staining shown in Figure 2 (top) was $1.45 \pm 0.02$, or less than $2 \%$.

\section{Results}

Effect of $\mathrm{KCl}$ application. Recurrent SD began 1-3 min after application of $\mathrm{KCl}$ to parietal cortex (Fig. 3, middle). Initial experiments $(n=6)$ were performed using $1 \mathrm{M} \mathrm{KCl}$. This resulted in $21 \pm 7$ SDs (range, 15-33; data are listed here and throughout as mean \pm standard deviation and range) over the 3-hr exposure period. Later, $3 \mathrm{M} \mathrm{KCl}$ was used $(n=9)$, but no significant ( $p$ $>0.8)$ increase in SDs was seen ( $22 \pm 8$; range, 16-33). Therefore, results from application of $\mathrm{KCl}$ were combined in to a single group. Application of 1 or $3 \mathrm{M} \mathrm{NaCl}$ to contralateral cortex never induced SD.

Recurrent SDs caused an increase in neocortical GFAP staining (Fig. 3, top; see also Table 2). $\mathrm{KCl}$ induced a more extensive but focal area of tissue necrosis at the application site than did an equivalent molarity of $\mathrm{NaCl}$. This conclusion is based on results from brains of two experimental animals that were embedded in paraffin and $6-\mu \mathrm{m}$ brain sections stained with hematoxylin and eosin. Focal brain injury was limited to the most caudal brain sections where 1 or $3 \mathrm{M}$ saline gels were applied (Fig. 4). Furthermore, as previously reported (Nedergaard and Hansen, 1987), recurrent SD did not induce any irreversible brain injury in any of the more rostral brain sections (Fig. 4). The two most caudal brain sections of each series (at -4 and $-5 \mathrm{~mm}$ from bregma) were excluded from data analyses to lower the chance that $\mathrm{KCl}$-induced injury (and associated gliosis) would 

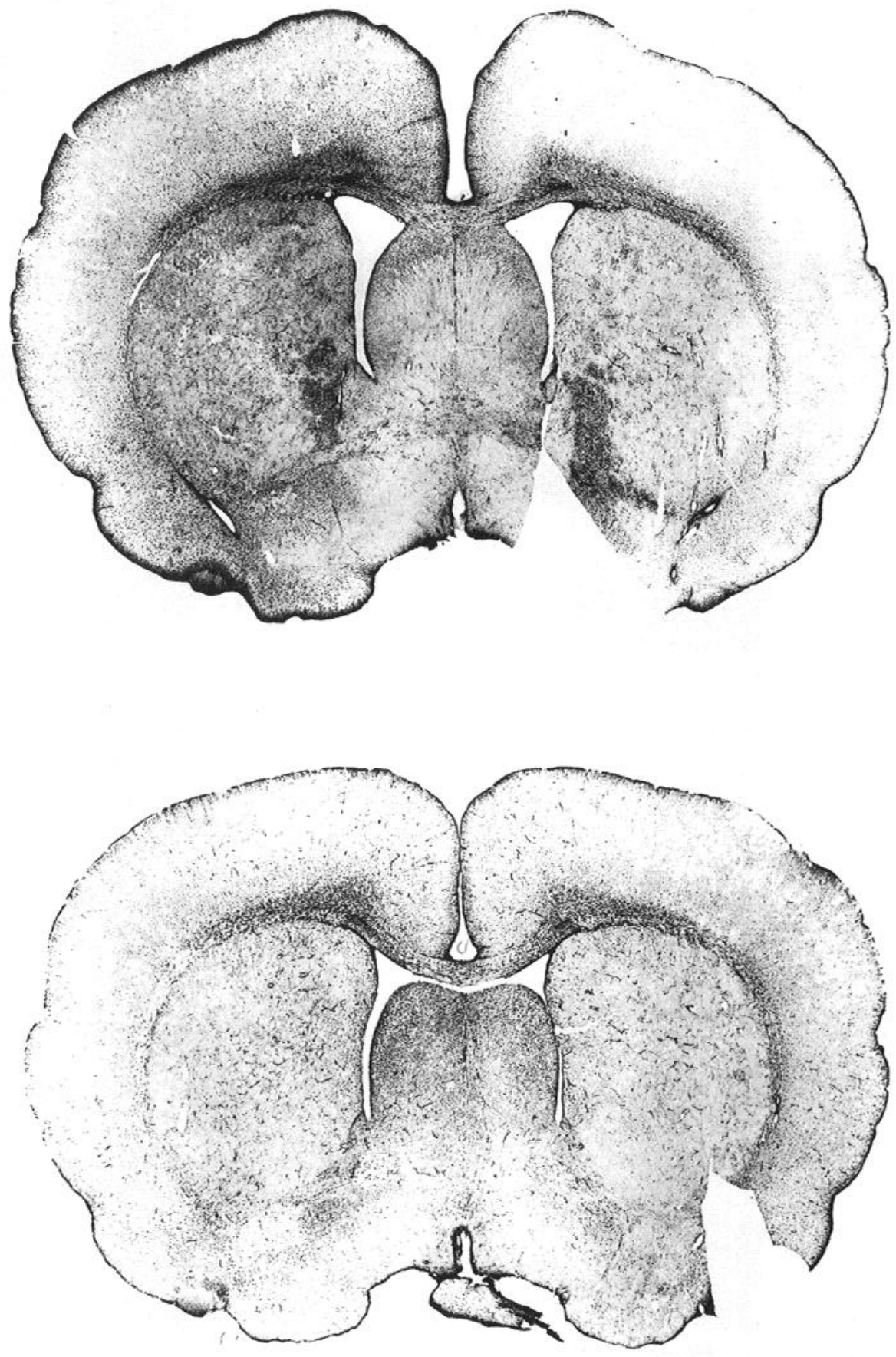

Figure 1. Bright-field photomicrographs of GFAP staining. Image from a frontal coronal section showing GFAP staining in experimental (left $)$ and control (right) neocortices at low power (top). $\mathrm{KCl}$ was applied to the left parietal cortex and $\mathrm{NaCl}$ to the right parietal cortex for 3 ir while DC signals were simultaneously monitored from both frontal cortices. No SD occurred in the right, while in this example, 37 SDs occurred in the left neocortex. Animals were allowed to recover for $48 \mathrm{hr}$ and then were processed for GFAP visualization by a peroxidase-anti-peroxidase staining technique. Coronal section from normal animal is shown for comparison (bottom). These images were used to produce color-coded images in Figure 3. bias results, though no such effect was apparent in the data. Seven brain sections ( +3 to $-3 \mathrm{~mm}$ from bregma) from eight experimental animals were used to show that recurrent SD produced an increase in neocortical GFAP staining intensity of $43 \%$ ( $t=9.92 ; n=8 ; p<10^{-4}$; Fig. 3 , top). Here, the $p$ value is based on the comparison of the log-ratio of left/right staining to 0 . Control animals (Fig. 3, bottom; $n=4$ ) showed no significant difference $(t=-0.73 ; n=4 ; p>0.5)$ from 0 in the log-ratio of staining.

In order to determine the permanency of neocortical, SDinduced increase in GFAP staining, we examined seven additional animals that were allowed to survive for $1-4$ weeks after exposure to $3 \mathrm{hr}$ of unilateral, neocortical SD. Analogous to the findings above, no trend was seen in differences from one brain section to the next, and section-to-section deviations were small compared to the mean of all sections for a given rat. Therefore, data were treated as done previously. Because only one or two animals were in each different survival group, the coefficient of variation in staining ratio was approximately the same at each time point. Furthermore, as with results from acute experiments (see above), no significant additional GFAP difference between neocortices was seen when the most caudal brain sections $(-4$ and $-5 \mathrm{~mm}$ from bregma) were included in analyses. Nonetheless, to reduce any bias that injury from the application of 

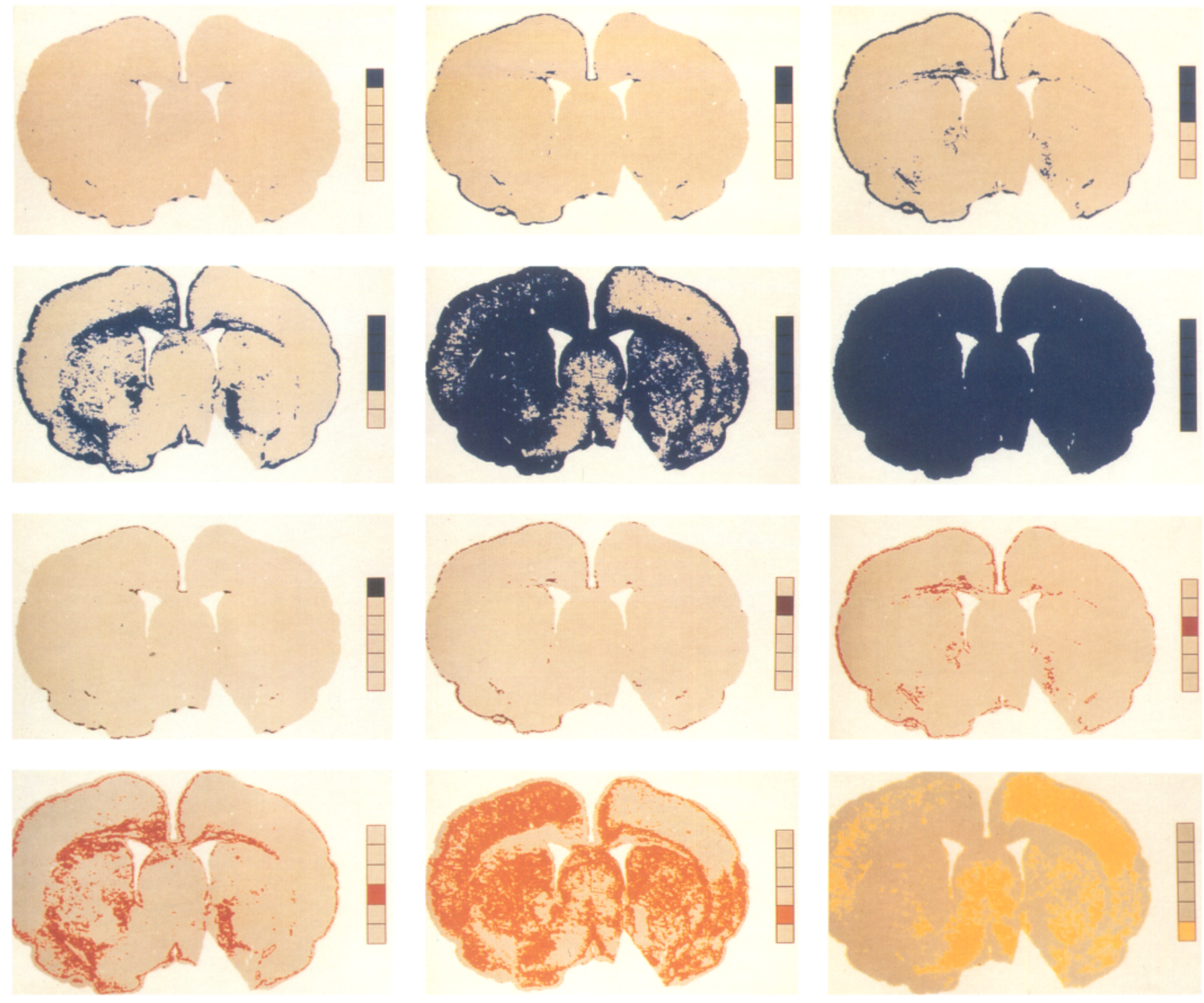

Figure 2. GFAP image analysis protocol. Coronal rat brain sections were analyzed with a computer-based system for their relative neocortical (left, experimental, compared to right, control) area of GFAP staining. Staining was measured on a linear gray scale (illustrated by two upper rows of blue color-coded images) from a darkest value threshold, where GFAP visualization began (first row, first image) to a lightest value threshold, where GFAP visualization reached saturation in the lighter-stained neocortex (second row, third image). This gray scale range was divided into six equally sized increments (shown as an accumulative increase in blue area above and with corresponding individual incremental areas in the two lower rows). To enhance visualization, six incremental areas of GFAP staining were color coded (two lower rows). Beginning with darkest shading (third row, first image; shaded with dark brown and reflecting the most intense GFAP staining), each progressively less intense incremental area was shaded a different color with the lightest intensity of GFAP staining shaded orange (fourth row, third image). Most of the GFAP area visualized occurred within the first three lighter zones. These individual areas were given a relative weighting factor to reflect their differences in staining intensity: The darkest area was multiplied by 6 , and each progressively lighter increment was multiplied by either $5,4,3,2$, or 1 , respectively. Then weighted areas were added together, and the resultant sum was normalized by dividing this value by the total area measured for a given cortical side. Finally, experimental-side values were divided by the corresponding control-side values, and these ratios were averaged for the nine sections measured for each animal. Thus, a single number was generated for each animal, which gave a weighted, normalized, and relative estimate of the area of GFAP staining in the experimental neocortex as compared to the control cortex.

$\mathrm{KCl}$ might include, these latter two sections were excluded from statistical analyses.

Recurrent SD caused a highly significant, but transient, increase in neocortical GFAP staining as compared to contralateral neocortices at 1 week ( $22 \%$ increase; $n=2 ; p<0.003), 2$ weeks ( $14 \%$ increase; $n=2 ; p<0.005), 3$ weeks ( $7 \%$ increase; $n=2 ; p<0.089)$, and 4 weeks ( $5 \%$ increase; $n=1 ; p<0.259$ ). Most of the reduction in significance in GFAP left-to-right difference occurred between weeks 2 and 3 . Although no significant increase in GFAP staining was evident at weeks 3 and 4, only positive deviations were observed at these times; that is, left (experimental) staining was always slightly greater than that found on the right (control) side. This suggests that an effect from SD may still be present in brain from animals that survived 3 and 4 weeks. However, if such effects are present, they are not large enough to be detected by comparisons based on such a small number of rats.

Effect of inhibition of $S D$ on associated increase in GFAP 

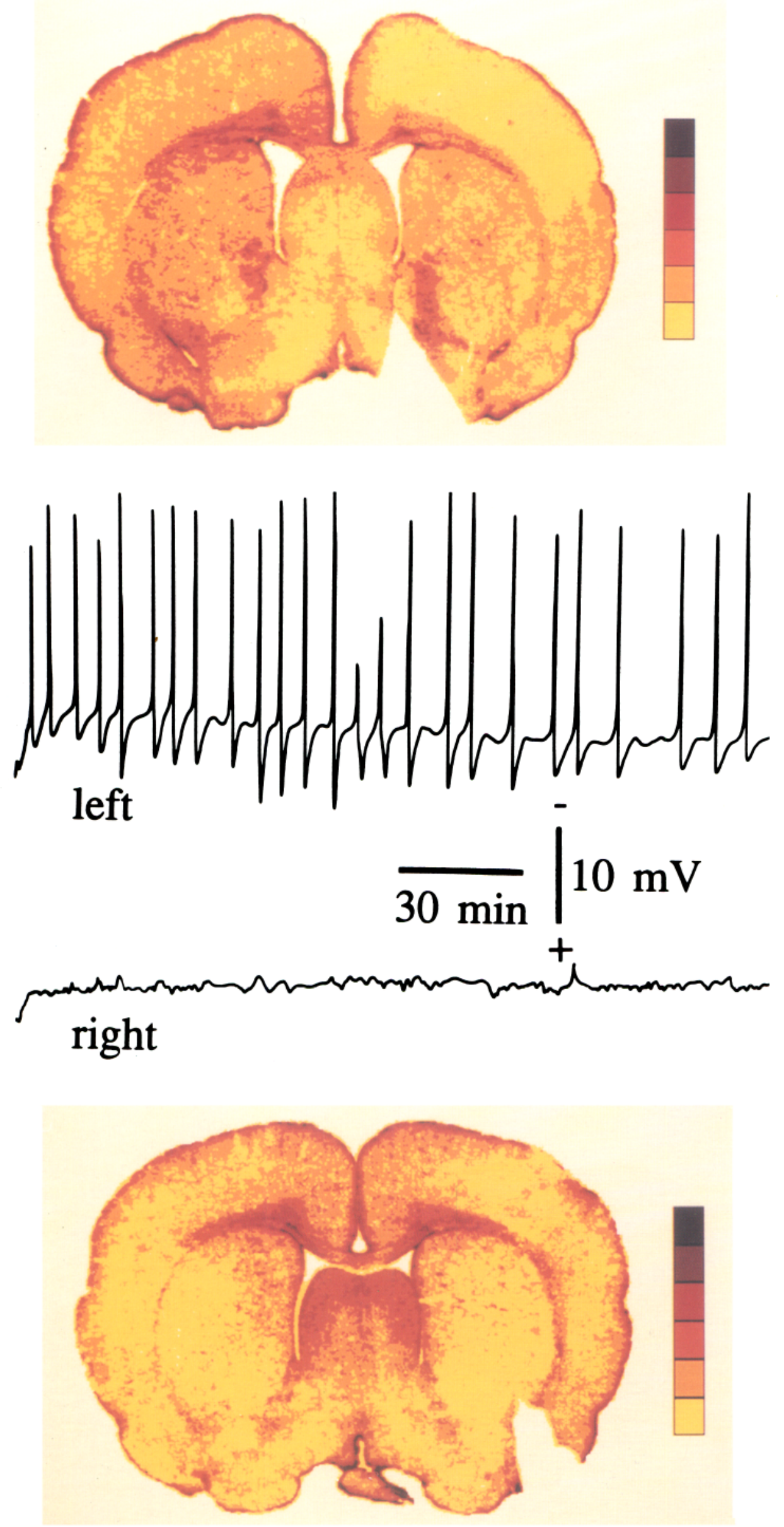

Figure 3. Color-coded GFAP staining profile and evidence for unilateral recurrent SDs from a single animal. The top color-coded image shows relative GFAP staining intensities (color bar is described in Fig. 2; darkest color indicates darkest staining). Left neocortex is shown on left. Associated DC signals are shown in the middle traces. Twentyfive SDs occurred in the left neocortex. while no SDs occurred in the right neocortex. Hence, recurrent SD produces a significant $\left(p<10^{-4}\right), 43 \%$ increase in GFAP staining compared to control contralateral cortices, which experienced no SDs $(n=8)$. For comparison, the bottom image shows color-coded GFAP staining from a normal animal. 

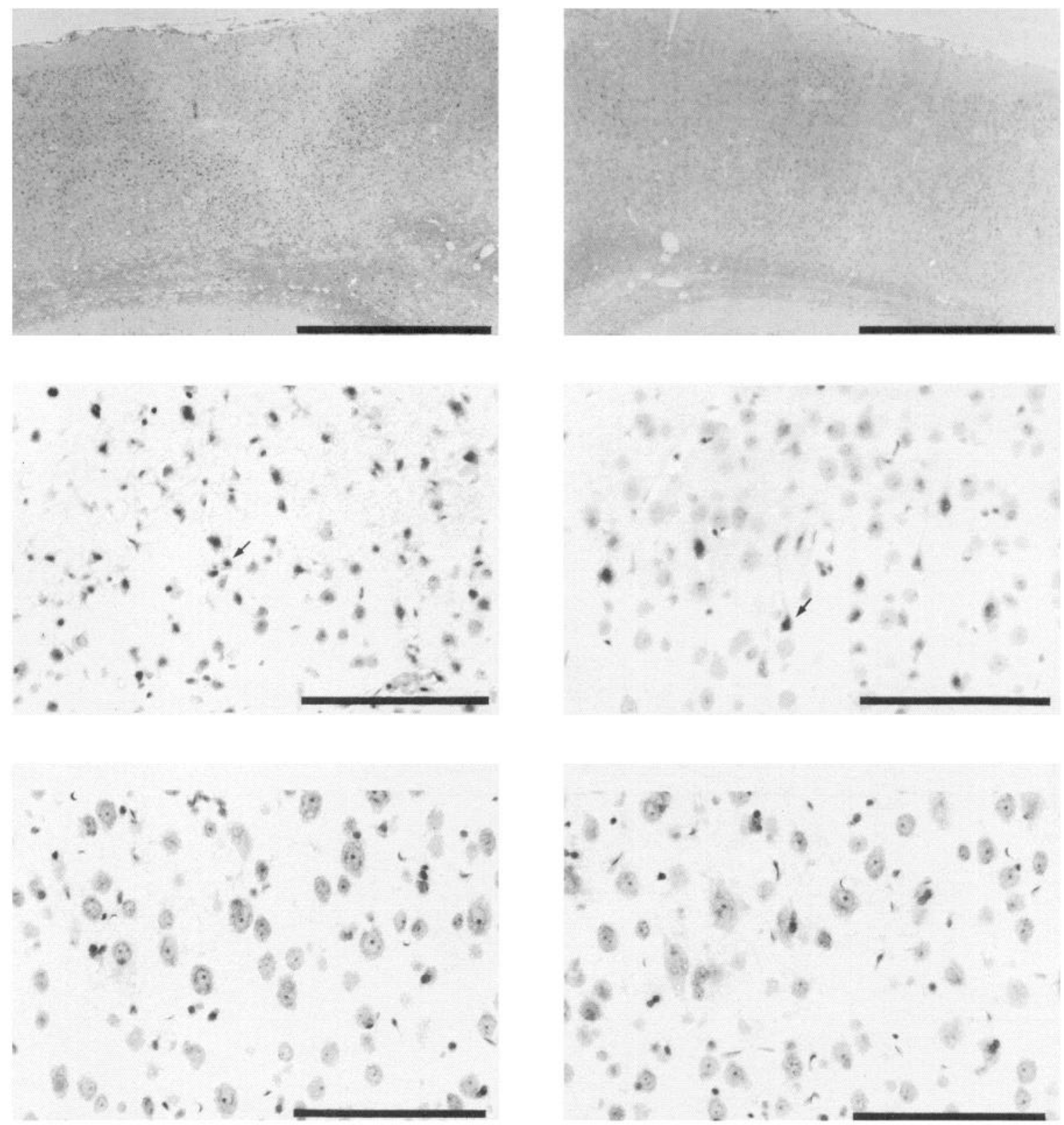

Figure 4. Hematoxylin- and eosin-stained brain sections. $\mathrm{KCl}$ induced coagulation necrosis, as indicated by wedge-shaped area of reduced staining (upper left). Most neurons within this area showed shrunken eosinophilic cytoplasm and pyknotic nuclei (center left, arrow). Nuclear fragmentation was evident, and nuclear debris was scattered in this zone. $\mathrm{NaCl}$, on the other hand, never produced coagulation necrosis (upper right). Instead, only "dark" neuronal changes are evident (center right, arrow). This change is consistent with reversible neuronal injury (see Duchen, 1984). KCl induced recurrent SD in the left hemisphere, and no evidence of reversible or irreversible neuronal injury is seen; section from left frontal cortex is shown (lower left). For comparative purposes, a right frontal section is also shown (lower right). No evidence of neuronal injury is evident. Both images show normal histological features of frontal neocortex. Scale bars: $500 \mu \mathrm{m}$, upper two micrographs; $50 \mu \mathrm{m}$ for remaining micrographs.

staining. SD from $\mathrm{KCl}$ exposure was prevented by modulation of inspired gasses to produce a state of combined hyperoxia and hypercarbia (Fig. 5). The electrophysiologic observations in all six animals were similar. Blood gas variables are shown in Table 1. Initial experiments $(n=3)$ were performed using $1 \mathrm{M} \mathrm{KCl}$ to induce SD. This stimulant resulted in no SD during the 3-hr exposure period. Similarly, no SD occurred in contralateral hemispheres exposed to $1 \mathrm{M} \mathrm{NaCl}$. Later experiments $(n=3)$ used $3 \mathrm{M} \mathrm{KCl}$ and $\mathrm{NaCl}$. Under these latter conditions, $\mathrm{KCl}$ caused $5 \pm 2$ (range, 2-6) SDs with no SDs occurring in the $\mathrm{NaCl}$-exposed cortices. Because of the low number of SDs that occurred, GFAP staining data were grouped into one population. This group $(n=6)$ showed that the induction of combined hyperoxia and hypercarbia reduced the SD-induced increase in GFAP staining so that no significant difference occurred between neocortical "experimental" and contralateral "control" staining (7\% increase; $t=1.61 ; n=6 ; p>0.200$; Fig. 5 , Table 2). The inspiratory gasses of another group $(n=3)$ of rats were modified to only include hyperoxia (Table 1). Under these conditions (data not shown), a significant difference ( $11 \%$ increase; $t=4.76$; $n=3 ; p>0.050$ ) was still evident between experimental and control neocortices (Table 2). However, hyperoxia did provide 

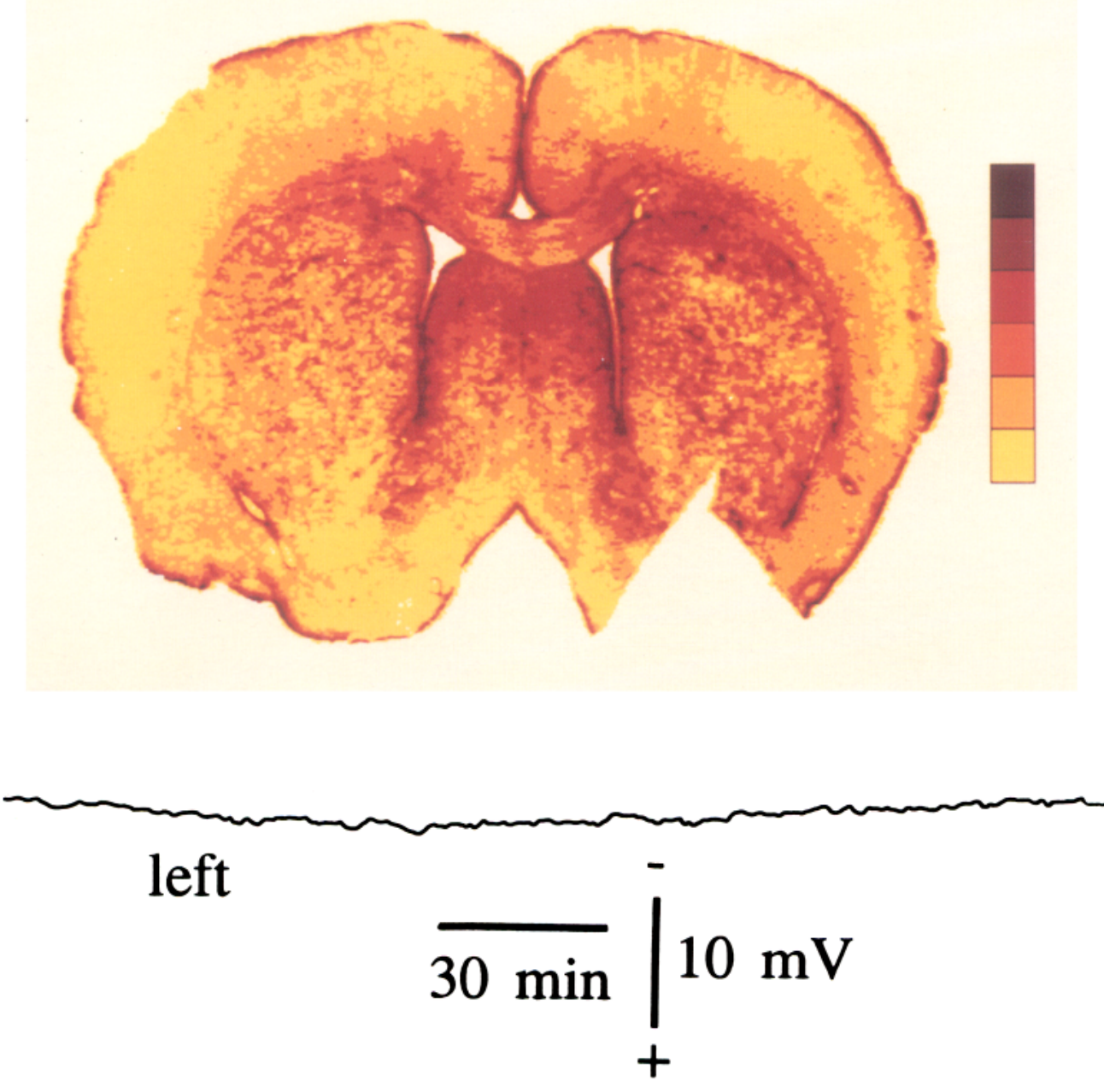

Figure 5. The effect of a combined increase in inhaled $\mathrm{O}_{2}$ and $\mathrm{CO}_{2}$ on GFAP staining and SD. After electrodes were implanted into frontal cortices to record DC signals, inhaled gasses were first altered to $100 \% \mathrm{O}_{2}$ and then, 5-10 min later, to include a progressively increasing amount of inhaled $\mathrm{CO}_{2} . \mathrm{CO}_{2}$ was increased and then adjusted to keep arterial $\mathrm{CO}_{2}$ tension between 100 and 120 torr. Inspired halothane remained constant at $1.0-2.0 \%$ of inhaled gasses. $\mathrm{KCl}$ was then applied to the left and $\mathrm{NaCl}$ to the right parietal cortex for 3 hr. No SDs were seen in this example (bottom). Color-coded GFAP staining is shown in the top (darkest color indicates darkest staining). Notice that in this example there is less intense GFAP staining in the experimental neocortex (left neocortex is on left) exposed to $\mathrm{KCl}$ than in the right neocortex exposed to $\mathrm{NaCl}$. However, in six animals exposed to this modification of inhaled gasses, a nonsignificant $(p>0.20), 7 \%$ increase in GFAP staining was evident in left as compared to right neocortices. some reduction in the number of SDs induced by $\mathrm{KCl}(3 \pm 1)$ and intensity of SD-induced GFAP staining difference (from $43 \%$ to $11 \%$ ).

The above results with regard to combined hyperoxia and hypercarbia were arrived at by exclusion of data from the two most caudal brain sections, which correlate with the area where saline gels were applied to the exposed cortices. Including these sections in the analysis produced virtually no change in the results. This finding and the fact that five out of six rats had log-ratios of GFAP staining greater than 0 indicated that hyperoxia and hypercarbia significantly reduced, but did not totally eliminate, an increase in GFAP staining near $\mathrm{KCl}$-injury sites.

When the two most caudal brain sections from each animal group were analyzed separately, it became evident that com-

Table 1. Blood physiologic variables at onset of SD

\begin{tabular}{llllllll}
$\begin{array}{l}\text { Animal } \\
\text { group }\end{array}$ & $\mathrm{pH}$ & $\begin{array}{l}\mathrm{PaCO}_{2} \\
\text { (torr) }\end{array}$ & $\begin{array}{l}\mathrm{PaO}_{2} \\
\text { (torr) }\end{array}$ & $\begin{array}{l}\text { Blood } \\
\text { pressure } \\
\text { (mm Hg) }\end{array}$ & $\begin{array}{l}\text { Hemat- } \\
\text { ocrit } \\
(\%)\end{array}$ & $\begin{array}{l}\text { Glucose } \\
(\mathrm{mm})\end{array}$ & $\begin{array}{l}\text { Temper- } \\
\text { ature } \\
\left({ }^{\circ} \mathrm{C}\right)\end{array}$ \\
\hline $\begin{array}{l}\mathrm{KCl} \text { alone }(n=15) \\
\begin{array}{l}\text { Hypercarbia and } \\
\text { hyperoxia } \\
(n=6)\end{array}\end{array}$ & $7.30 \pm 0.02$ & $60 \pm 4$ & $119 \pm 19$ & $110 \pm 8$ & $46 \pm 4$ & $4.9 \pm 1.2$ & $36.9 \pm 0.4$ \\
$\begin{array}{l}\text { Hyperoxia } \\
(n=3)\end{array}$ & $7.06 \pm 0.04$ & $115 \pm 11$ & $380 \pm 76$ & $101 \pm 7$ & $46 \pm 5$ & $5.2 \pm 1.6$ & $37.0 \pm 0.4$ \\
& $7.30 \pm 0.03$ & $60 \pm 5$ & $423 \pm 51$ & $120 \pm 0$ & $48 \pm 5$ & $4.2 \pm 0.9$ & $37.1 \pm 0.2$ \\
\hline
\end{tabular}



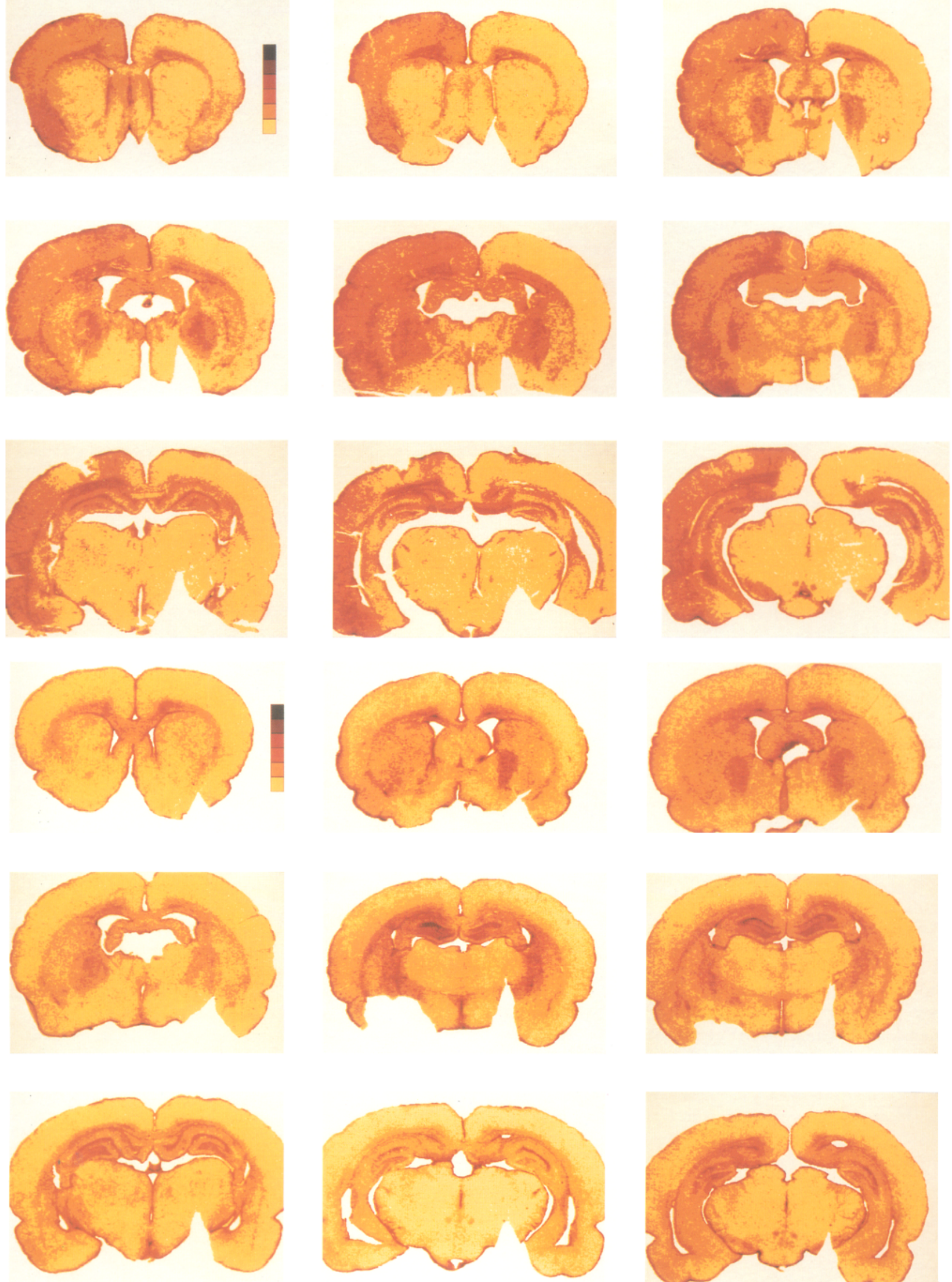

Figure 6. Effect of a combined increase in $\mathrm{O}_{2}$ and $\mathrm{CO}_{2}$ on SD- and $\mathrm{KCl}$-induced increase in GFAP staining. The dramatic reduction of the increase in GFAP staining from SDs caused by exposure to elevated $\mathrm{O}_{2}$ and $\mathrm{CO}_{2}$ is further emphasized by a nearly similar reduction in GFAP staining around the necrotic zone where $\mathrm{KCl}$ was applied. The full nine brain sections (three upper rows) from the animal whose results are shown in Figure 3 are presented. In this experiment, 25 SDs were seen in the left neocortex, and none in the right. Notice that GFAP staining is more intense in 
Table 2. Neocortical GFAP staining differences (experimental compared to contralateral control)

\begin{tabular}{llll} 
Animal group & Number of SDs & Caudal two sections $^{\circ}$ \\
\hline $\begin{array}{l}\text { KCl alone } \\
(n-8)\end{array}$ & $24 \pm 8$ & $0.358 \pm 0.102$ & $0.372 \pm 0.053$ \\
& & $43 \%$ increase & $45 \%$ increase \\
Hypercarbia & $5 \pm 2$ & $p<10^{-4}$ & $p<10^{-6}$ \\
and hyperoxia & & $0.0635 \pm 0.097$ & $0.079 \pm 0.059$ \\
$(n=6)$ & $3 \pm 1$ & $7 \%$ increase & $6 \%$ increase \\
Hyperoxia & & $p>0.20$ & $p>0.10$ \\
$\quad(n=3)$ & & $0.108 \pm 0.040$ & $0.099 \pm 0.030$ \\
& & $11 \%$ increase & $10 \%$ increase \\
\end{tabular}

${ }^{a}$ Mean \pm standard deviation.

${ }^{b}$ Results are listed as mean log-ratio \pm standard deviation, $\%$ increase, and $p$ value.

bined hyperoxia and hypercarbia also reduced the intensity of GFAP staining around the neural tissue damaged from application of saline gels (Table 2). Figure 6 shows the typical leftto-right staining differences in all nine brain sections from an animal that produced 25 SDs (Fig. 6, top) and the absence of such differences in an animal that produced only six SDs (Fig. 6 , bottom) because of hyperoxia and hypercarbia. The reduction in staining difference at the caudalmost brain sections is evident. The percent increase of the staining difference (Table 2 ) in the zone ( -4 and $-5 \mathrm{~mm}$ from bregma) where concentrated saline gels were applied was $45 \%$ when $\mathrm{KCl}$ (experimental side) was compared to $\mathrm{NaCl}$ (control side). Exposure to combined hyperoxia and hypercarbia reduced this increase to $6 \%$, and hyperoxia alone reduced the change to $10 \%$.

\section{Discussion}

Past studies have concluded that activity-induced modulation of the brain cell microenvironment could ultimately influence normal neuronal function (Cserr, 1986). The present data suggest that physiologic changes accompanying SD may also effect the induction of increased GFAP staining within astrocytes. Our measurements show that recurrent SD is associated with a highly significant and transient increase in GFAP staining that returns to near normal after 2-3 weeks. Furthermore, this SD-induced change in GFAP staining can be inhibited when SD is prevented by combined exposure to hyperoxia and hypercarbia. Finally, such a manipulation of inhaled gasses is even capable of significantly retarding any GFAP staining increase dependent upon tissue necrosis from exposure to concentrated $\mathrm{KCl}$.

GFAP is a $50,000-\mathrm{Da}$ protein that forms the major constituent of the cell-type-specific intermediate filament (Lazarides, 1982) found in astrocytes (and related cell types; for review, see Eng, 1980). Immunohistochemical staining for this protein is now used to characterize brain cells as astrocytic (Bignami and Dahl, 1976; Latov et al., 1979). After brain injury, astrocytes commonly undergo transformation to reactive species that have the histological characteristics of hypertrophy and hyperplasia
(Duchen, 1984). Expectedly, changes in size and number of these cells are commonly accompanied by an increase in GFAP staining, which has come to be recognized as an additional fundamental characteristic of reactive astrocytes (Bignami and Dahl, 1976; Latov et al., 1979). An increase in GFAP staining does not necessarily correlate to an increase in GFAP content. Aquino et al. (1988) have shown that, shortly after induction of experimental allergic encephalitis (EAE) in Lewis rats, GFAP staining rises while GFAP content remains unchanged. Only later in the evolution of this disorder does GFAP staining and content show a positive correlation. This discrepancy between immunohistochemical and biochemical measurements of GFAP is believed to result from a physical rearrangement of intermediate filaments caused by astroglial swelling (Aquino et al., 1988). As a result, more antigenic determinants may be exposed for reaction with GFAP antibodies to produce increased staining for this protein (Aquino et al., 1988). A similar phenomenon could account for the rise in GFAP staining seen after recurrent $\mathrm{SD}$, because astrocytes in vivo probably swell with depolarization (Van Harreveld, 1982; Kraig and Petito, 1989). Whether SD caused changes in GFAP biochemistry or actually induced an increase in GFAP content remains unknown.

Petito and colleagues (Petito et al., 1990) have shown that global ischemia, which results in the selective loss of vulnerable neurons, induces two forms of reactive astrocytosis. For example, astrocytes adjacent to CA1 hippocampal pyramidal cells that arc irreversibly injurcd by global ischemia show an incrcase in GFAP staining associated not only with hypertrophy, but also with hyperplasia. On the other hand, within neocortex, only scattered neocortical neurons in layers III, V, and VI are lost laterally, while medially, no neurons are destroyed. Astrocytes in paramedian neocortex undergo an increase in GFAP staining that is only associated with hypertrophy after global ischemia. A similar dual pattern of reactive astrocytosis is seen after needle-stab injury (Takamiya et al., 1988). Needle stab increases GFAP staining thoughout the associated neocortex, but secondary double labeling by ${ }^{3} \mathrm{H}$-thymidine autoradiography shows

$\leftarrow$

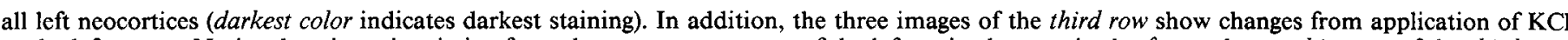

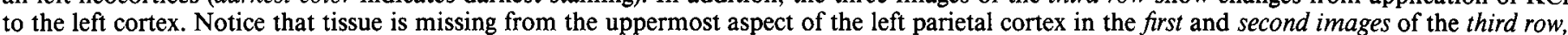

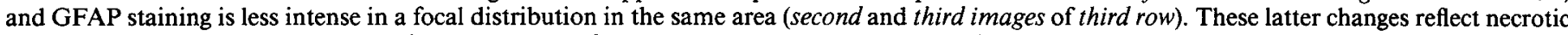

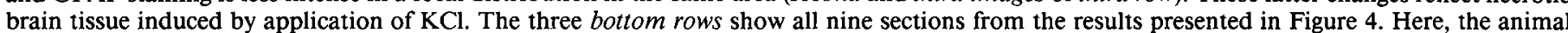

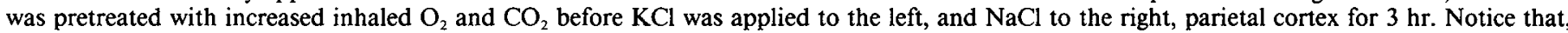

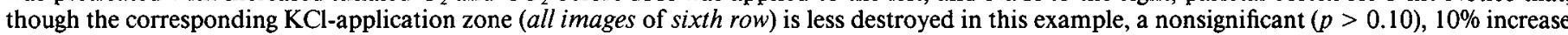

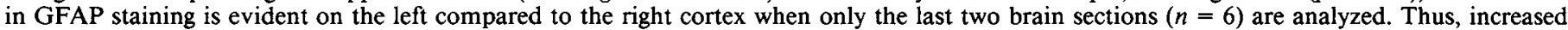
$\mathrm{O}_{2}$ and $\mathrm{CO}_{2}$ provided some protection against any increased GFAP staining from $\mathrm{KCl}$-induced brain necrosis. 

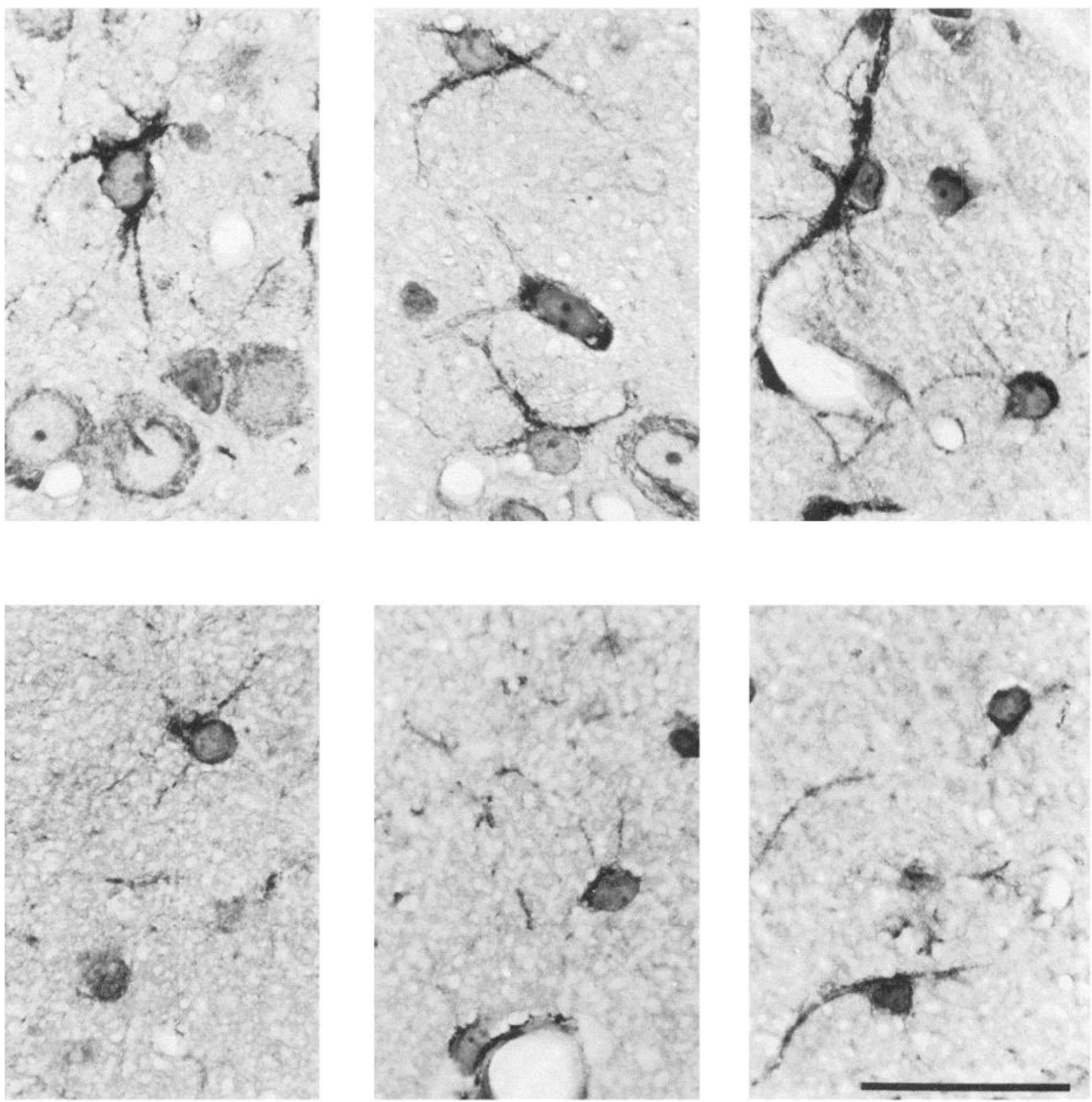

Figure 7. Toluidine blue- and GFAP-stained thin sections showing astrocytic hypertrophy after SD. The three upper images show thickened and more extensive GFAP processes of hypertrophied astrocytes from cortex that underwent 25 SDs. The three lower images show astrocytes from contralateral neocortex. The latter cells are smaller and have less shorter and thinner processes than those from the SD hemisphere. Scale bar, $50 \mu \mathrm{m}$.

that proliferation of astrocytes occurs only in the molecular layer and in gray and white matter adjacent to the wound. No astrocytic mitosis is seen farther away from the stab injury in neocortical layers II-VI. SD induced an increase in GFAP staining. This means that the area of GFAP staining (as well as the intensity of this staining) increased after SD (e.g., see Fig. 1). It is likely that SD also induces a hypertrophy of astrocytes. Indeed, preliminary data indicate that SD causes an enlargement of astrocytes. Thin $(1-\mu \mathrm{m})$ sections were cut by a simple cooling procedure (Junqueira et al., 1989) from a paraffin block used for hematoxylin and eosin staining and are shown in Figure 7. Sections were processed for GFAP staining and then also stained with toluidine blue. Although systematic measurements were not made, more and hypertrophied astrocytes were evident in the neocortex, which underwent recurrent $\mathrm{SD}$, than in the con- tralateral control (Fig. 7). Thus, the increase in GFAP staining intensity and area and the hypertrophy of astrocytes associated with SD resemble those changes seen in these cells within paramedian neocortex after global ischemia.

Neuronal necrosis has been considered to be essential for the induction of reactive astrocytosis. However, in light of the evidence provided by Petito and colleagues (Petito et al., 1990) and Takamiya and colleagues (Takamiya et al., 1988), as well as that presented in this article, such necrosis is not required for the reactive changes, which consist of hypertrophy and increased GFAP staining. More physiologic processes need to be considered as sufficient stimuli for a transient metamorphosis of susceptible astrocytes into reactive species. Specific mechanisms capable of triggering this transformation of astrocytes are unknown. However, the intimate involvement of astrocytes in 
ion (Walz, 1989) and neurotransmitter (Kimelberg, 1988; Hertz and Schousboe, 1986) homeostasis of CNS tissue suggests these phenomena as more obvious initial areas to examine for potential stimuli.

Virtually complete depolarization is one factor common to astrocytes during SD or brain ischemia. Changes in interstitial $\left[\mathrm{K}^{+}\right]$(and, as a result, $\left[\mathrm{K}^{+}\right]_{i}$ ) have long been considered as capable of modulating astrocytic metabolism and proliferation under normal and pathological conditions (for review, see Reichenbach, 1989). Furthermore, depolarization (by $\mathrm{K}^{+}$) itself has been considered a key signal for proliferation (Stillwell et al., 1973). Finally, in cultured $3 T 3$ fibroblasts, deoxyribonucleic acid synthesis is highly dependent on $\mathrm{K}^{+}$and only occurs when $\mathrm{K}^{+}$i exceeds $90 \mathrm{~mm}$ (Lopez-Rivas et al., 1982). If $\mathrm{K}^{+}$has the same regulatory ability in astrocytes as it does in cultured fibroblasts, increased GFAP staining seen in these cells after SD may be due to fluctuations in $\left[\mathrm{K}^{+}\right]_{i}$ because the activity of this ionic species climbs to 100-120 mM during SD (R. P. Kraig and C. Iadecola, unpublished observations). Fluctuations in $\left[\mathrm{K}^{+}\right]_{i}$ could influence astrocytic metabolism because of concomitant changes in $\mathrm{pH}_{i}$.

Astrocytes in vivo increase their $\mathrm{pH}_{i}$ roughly in proportion to the degree of their depolarization during evoked neuronal activity, SD (Chesler and Kraig, 1987, 1989), and global ischemia (under normoglycemic conditions; Kraig and Petito, 1989). During SD, astrocytes are almost completely depolarized, and their $\mathrm{pH}_{i}$ rises from 7.0 to $7.4-7.6$. A rise in $\mathrm{pH}_{i}$ typically correlates with an increase in metabolic activity (including protein synthesis, glycolysis, and proliferation), while a reduction in $\mathrm{pH}_{i}$ often accompanies a reduction in vital cellular processes such as cellular dormancy (for review, see Busa and Nucitelli, 1984). If variations in $\mathrm{pH}_{i}$ serve a modulatory function in astrocytes, perhaps any inhibition of increased GFAP staining that occurred near the necrotic zone where $\mathrm{KCl}$ was applied resulted from a cellular acidosis caused by hypercarbia. Why hyperoxia alone should also reduce $\mathrm{KCl}$ - or SD-induced incrcase in GFAP staining is unclear but could relate to an enhanced capacity for $\mathrm{K}^{+}$ regulation provided by the availability of more $\mathrm{O}_{2}$ than normal. Support for this conjecture comes from our preliminary experiments (Kraig and Chesler, 1988), which indicate that modulation of glucose supply to brain is one determinant of the frequency of $\mathrm{SD}$ that results from application of $\mathrm{KCl}$ to remote neocortex.

If ionic changes (i.e., $\left[\mathrm{K}^{+}\right]_{i}$ and $\mathrm{pH}_{i}$ ) that accompany astrocytic depolarization prove to be instrumental for initiation of increased GFAP staining, then the required magnitude of the ionic changes that bring about a difference in staining intensity may be smaller than that seen during SD. This conclusion stems from the fact that SDs (and presumably the large ionic changes associated with astrocytic depolarization from it) do not extend past the gray matter zone through which the phenomenon propagates (Bures et al., 1974), yet increased GFAP staining is clearly evident in the basal ganglia ipsilateral to neocortex that underwent SD as compared to the contralateral, control area (Figs. 3; 5 , top). Because neuronal afferents from the neocortex should depolarize and thus release $\mathrm{K}^{+}$in the basal ganglia during neocortical SD, adjacent astrocytes in this subcortical structure are likely to undergo ionic changes closer to that seen from evoked neuronal activity. Stimulation of the cortical surface causes astrocytic $\left[\mathrm{K}^{+}\right]_{i}$ to rise by $10-15 \mathrm{~mm}$ (Kraig and C. Iadecola, unpublished observations) and astrocytic $\mathrm{pH}_{i}$ to rise by approximately 0.1 pH (Chesler and Kraig; 1987, 1989). Recurrent photic stimulation for $3 \mathrm{hr}$ does not result in increased GFAP staining in the optic tectum (Kraig and Jaeger, 1990). Thus, a lower limit to the magnitude (and duration?) of astrocytic depolarization needed to alter GFAP staining remains to be defined.

Neocortical afferents to the basal ganglia are largely glutaminergic (Ottersen and Storm-Mathisen, 1984). Thus, depolarization of these afferents by neocortical SD is likely to release glutamate to the interstitial space of the ipsilateral basal ganglia. Interstitial glutamate is avidly metabolized by astrocytes (Hertz and Schousboe, 1986). Perhaps exposure of susceptible astrocytes to glutamate is a stimulus capable of transforming these cells in vivo into hypertrophied cells with increased GFAP staining.

The ease with which SD can be elicited in vivo and in in vitro brain slice preparations and the reproducible and stereotypic nature of the phenomenon suggest that it may be a unique, physiologic perturbation with which to begin to elucidate some of the cellular mechanisms of reactive astrocytosis.

\section{References}

Aquino DA, Chiu F-C, Brosnan CF, Norton WT (1988) Glial fibrillary acidic protein increases in the spinal cord of Lewis rats with acute experimental autoimmune encephalomyelitis. J Neurochem 51:10851096.

Bignami A, Dahl D (1976) Astroglial response to stabbing. Immunofluorescence studies with antibodies to astrocyte-specific protein (GFA) in mammalian and submammalian vertebrates. Neuropathol Appl Neurobiol 2:99-1 10.

Bretag AH (1969) Synthetic interstitial fluid for isolated mammalian tissue. Life Sci 8:319-329.

Bures J, Buresova O, Krivanek J (1974) The mechanism and applications of Leao's spreading depression of electroencephalographic activity. New York: Wiley.

Busa WB, Nucitelli R (1984) Metabolic regulation via intracellular pH. Am J Physiol 246:R409-R438.

Chesler M, Kraig RP (1987) Intracellular $\mathrm{pH}$ of astrocytes increases rapidly with cortical stimulation. Am J Physiol 253:R666-R670.

Chesler M, Kraig RP (1989) Intracellular pH transients of mammalian astrocytes. J Neurosci 9:2011-2019.

Cserr HF, ed (1986) The neuronal microenvironment. Ann NY Acad Sci 481.

Duchen LW (1984) General pathology of neurons and glia. In: Greenfield's neuropathology (Adams JH, Corsellis JAN, Duchen LW, eds), pp 1-52. New York: Wiley.

Eng LF (1980) The glial fibrillary acidic (GFA) protein. In: Proteins of the nervous system (Bradshaw RA, Schneider DM, eds), pp 85117. New York: Raven.

Gardner-Medwin AR (1981) Possible roles of vertebrate neuroglia in potassium dynamics, spreading depression and migraine. I Exp Biol 95:111-127.

Hertz L, Schousboe A (1986) Role of astrocytes in compartmentation of amino acid and energy metabolism. In: Astrocytes, Vol 2 (Federoff S, Vernadakis A, eds), pp 179-208. New York: Academic.

Jaeger CB (1988) Plasticity of astroglia: evidence supporting process elongation by "stretch." Glia 1:31-38.

Junqueira LCU, Silva DA, Torloni H (1989) A simple procedure to obtain one-micrometer sections of routinely embedded paraffin material. Stain Technol 64:39-42.

Kimelberg HK (1988) Glial cell receptors. New York: Raven.

Kimelberg HK, Ransom BR (1986) Physiological and pathological aspects of astrocytic swelling. In: Astrocytes, Vol 3, cell biology and pathology of astrocytes (Fedoroff S, Vernadakis A, eds), pp 129-158. New York: Academic.

Kraig RP, Chesler M (1988) Dynamics of volatile buffers in brain cells during spreading depression. In: Mechanisms of cerebral hypoxia and stroke (Somjen G, ed), pp 279-289. New York: Plenum.

Kraig RP, Jaeger CB (1990) Recurrent spreading depression increases GFAP staining. Soc Neurosci Abstr 16:351.

Kraig RP, Petito CK (1989) Interrelation of proton and volume regulation in astrocytes. In: 16th Princeton Research Conference, Cere- 
brovascular discases (Ginsberg MD, Dietrich WD, eds), pp 239-246. New York: Raven.

Latov N, Nilaver G, Zimmerman EA, Johnson WG, Silverman A-J, Defendini R, Cote L (1979) Fibrillary astrocytes proliferate in response to injury. Dev Biol 72:381-384.

Lazarides E (1982) Intermediate filaments: a chemically heterogeneous, developmentally regulated class of proteins. Annu Rev Biochem 51:219-250.

Lopez-Rivas A, Adelberg EA, Rozengurt E (1982) Intracellular $\mathrm{K}^{+}$ and the mitotic response of 3 T3 cells to peptide factors in serum free medium. Proc Natl Acad Sci USA 85:8727-8731.

Mize RR, Holdefer RN, Nabors LB (1988) Quantitative immunohistochemistry using an image analyzer. I. Hardware evaluation, image processing, and data analysis. J Neurosci Methods 26:1-24.

Nedergaard M, IIansen AJ (1987) Spreading depression is not associated with neuronal injury in rat brain. Brain Res 449:395-398.

Niggli V, Burger MM (1987) Interaction of the cytoskeleton with the plasma membrane. J Membr Biol 100:97-121.

Norenberg MD, Hertz L, Schousboe A, eds (1988) The biochemical pathology of astrocytes. New York: Liss.

Ottersen O, Storm-Mathisen J (1984) Neurons containing or accumulating transmitter amino acids. In: Handbook of chemical anat- omy, Vol 3. Classical transmitters and transmitter receptors in the CNS, Pt II (Bjorklund A, Hokfelt T, Kuhar MJ, eds), pp 141-246. New York: Elsevier.

Petito CK, Morgello S, Felix JC, Lesser MI (1990) The two patterns of reactive astrocytosis in postischemic brain. J Cereb Blood Flow Metab 10:850-859.

Reichenbach A (1989) Glia:neuron index: review and hypothesis to account for different values in various animals. Glia 2:71-77.

Snow RW, Taylor CP, Dudek FE (1983) Electrophysiological and optical changes in slices of rat hippocampus during spreading depression. J Neurophysiol 50:561-572.

Stillwell EF, Cone CM, Cone CD Jr (1973) Stimulation of DNA synthesis in CNS neurones by sustained depolarization. Nature 246:110 111.

Takamiya Y, Kohsaka S, Toya S, Otani M, Tsukada Y (1988) Immunohistochemical studies on the proliferation of reactive astrocytes and the expression of cytoskeletal proteins following brain injury in rats. Dev Brain Res 38:201-210.

Van Harreveld A (1982) Swelling of the Müller fibers in the chicken retina. J Neurobiol 13:519-536.

Walz W (1989) Role of glial cells in the regulation of the brain ion microenvironment. Prog Neurobiol 33:309-333. 